\title{
RADIO ET RÉCITS : DU CAS EMBLÉMATIQUE DES SPOTS PUBLICITAIRES À UNE RENAISSANCE
}

\author{
Frédéric Antoine ${ }^{1}$
}

Philippe et Mathilde forment un couple comme les autres. Longtemps, ils n'ont vécu qu'à deux, en compagnie sporadique de «belle-maman », alias la mère de madame, une femme particulièrement intrusive. Un jour, Mathilde est tombée enceinte, puis a accouché de Juliette. La famille s'est ensuite étendue à Régis, l'ami un peu benêt, avant que Mathilde ne retombe enceinte. Thibault est alors venu compléter la famille parfaite, tandis que Juliette, grandissant, s'est trouvé son premier amoureux : Thomas. De son côté, Belle-maman faisait de même, et introduisait dans le cercle familial son nouveau chevalier-servant : Gaston...

À l'heure où ces lignes sont écrites, l'auditeur des radios privées françaises ignore la suite des aventures qui attendent cette famille moyenne. Mais il se doute qu'elle connaîtra encore de nombreux

1 Frédéric Antoine est professeur au Département de communication de l'UCL

Recherches en communication, $\mathrm{n}^{\circ} 26$ (2006). 
rebondissements qu'il aura le loisir d'entendre, à de multiples reprises, au cours de pastilles radiophoniques d'une durée immuable de 30 secondes. Car cela fait des années que dure l'histoire de la «famille Leclerc ». Et cela fait tout autant de temps que ce nom de famille est à placer entre des guillemets, puisqu'il représente d'abord celui de l'enseigne commerciale que ces histoires sont chargées de promotionner : celle des hypermarchés Édouard Leclerc.

Les péripéties de la « famille Leclerc », racontées au quotidien sur les antennes, sont en effet... des spots publicitaires dont le trait de génie est d'avoir été conçu sur le mode de la sitcom radiophonique.

L'exemple ci-dessous, diffusé sur les ondes françaises en novembre 2006, manifeste la configuration présentée par la majeure partie des spots de ce type. En l'occurrence, il met en scène le mari, Philippe, et Régis, l'ami de la famille.

«- Je te dérange, Philippe?

- Je pars, Régis!

- Mais où pars-tu?

- Chez Leclerc, Régis. Ils remettent ça!

- Ils remettent quoi?

- $50 \%$ de pouvoir d'achat en plus.

- Encore! En ce moment?

— Oui mon bon, plus rien ne les arrête. Allez, fidèle Régis, joinstoi à ma quête!

- Oui, mais ta quête de quoi?

- Ben, des $50 \%$ de pouvoir d'achat en plus!

-Wouaaais!»

Tous les écrans radios pour les supermarchés Leclerc ne parodient pas les grands genres littéraires à la manière de celui qui a été reproduit ici, mais tous mettent en scène, et en situation de dialogue, deux des protagonistes de la «famille Leclerc » tels qu'ils ont été présentés ci-dessus.

\section{Le spot narratif}

On peut de la sorte relever que les spots publicitaires pour ces grands surfaces se distinguent du mainstream de la production du genre 
par plusieurs des composantes que laissait déjà entrevoir la notion de « sitcom radiophonique » précédemment évoquée : l'élément de feuilletonisation des contenus, leur mise en scène en respectant les trois règles de base de la tragédie classique et la matérialisation de ces contenus via un processus de personnification marqué.

Autrement dit, pour la partie qui nous intéresse ici et qui ne relève pas de la mécanique de la promotion de marque, ces spots publicitaires s'avèrent essentiellement conçus comme une suite diachronique de courts récits dialogués dans laquelle la narrativité et sa mise en œuvre séquentielle remplissent un rôle déterminant.

La mise en récit permet à l'auditeur de se figurer les personnages et d'inscrire leur échange de paroles en une situation.

L'évolution de récit au fil du temps, sur le mode du feuilleton, construit l'épaisseur des personnages, favorise leur familiarisation et celle des lieux dans lesquels ils évoluent.

Ces divers éléments façonnent le caractère sériel de l'œuvre radiophonique considérée, dont le «sitcomique » est manifesté par le ton de chacun des dialogues, traitant sur un mode badin de thèmes en liaison avec la banalité de l'existence : vie familiale et de couple, relations amicales ou filiales, envies féminines (d'enfant, de travail...), gourmandise masculine, mots d'enfants... et, bien sûr, célébration du consumérisme quotidien au travers de la permanence de l'évocation du culte des « courses chez Leclerc».

La récurrence de la présence de la «famille Leclerc » au sein des publicités pour la marque produit sur l'auditeur le même effet que celui engendré par le suivi répété de feuilletons télévisés : les personnages entrent dans les vies des récepteurs du message ${ }^{1}$. Les commentaires que l'on peut recueillir sur divers blogs glanés sur Internet en attestent.

Ainsi, par exemple :

«Hé bien moi je m’y suis attachée. Vraiment! Sont attendrissants !! Toutes leurs petites histoires. ,. Rhalala... Je crois que c'est les

1 Dans le cas la publicité Leclerc, cette proximité est renforcée par le fait que les personnages et les textes des messages ont été imaginés et sont écrits par le directeur de l'agence qui les produit, Philippe Gautier de l'agence Lollipop. 
seules pubs que j'écoute vraiment même si ça ne me fait pas plus aller au magasin dont ils vantent les mérites. La petite histoire du début... L'énoncé de la promo... Et le trait d'humour de la fin... J'vous dis la seule que j'écoute! Et pourquoi ? Peut être justement à cause de cette présence répétitive des personnages qui reviennent toujours. Ou alors j'en sais rien! ${ }^{1}$

$\mathrm{Ou}$ :

«Je n'ai pas la télé donc je vais parler de ce que j'entends, c'est à dire Mathilde et Philippe qui vantent les produits Leclerc. (...) Cela se présente sous forme de "tranche vie » d'un couple qui pourrait être le votre ou le mien : reproche, dispute, tendresse, amitié, fin de mois (plus un rond), fêtes diverses... Les deux protagonistes dialoguent et évoquent un produit et surtout la carte leclerc qui est une véritable obsession. Lui parle un peu trop vite à mon goût, c'est limite incompréhensible. En fait, j'ai écouter [sic] une émission à laquelle participaient Mathilde et Philippe et c'était vraiment sympas [sic] parce qu'ils sont quasi pareils que dans la pub surtout lui...Je crois d'ailleurs que ce sont leurs vrais prénom, Ils travaillent eux-même [sic] sur les textes. Elle est la fille de Sylvie Joly...

C'est vrai qu'il m'arrive de téléphoner à mon mari pour lui annoncer une super promo etc...

C'est parfois affligeant de voir son reflet à la radio... C'est tellement réel... $»^{2}$.

Leanaya, qui signe le message ci-dessus, membre de « La communauté shopping Ciao » depuis 2002, écrivait cela le 27 mai 2003, en titrant : «La pub Leclerc à la radio, c'est rigolo ». Et en ajoutant : «Nous sommes tous des Mathilde et Philippe en puissance. Et c'est bien là le drame. »

Un drame dans lequel la fidélisation par feuilletonisation du récit joue assurément un rôle primordial. D'autres publicités radiophoniques cultivent des éléments de même type, mais sans les entretenir avec autant de constance que la « famille Leclerc ».

1 (Blog Série 8 Groupe D! http://s8gd.midiblogs.com/archive/2006/05/index.html)

2 http://www.ciao.fr/E_Leclerc__Avis_512211 
En Belgique francophone, tel a par exemple été le cas de TéléSecours, service d'assistance à domicile pour personnes âgées, qui a imaginé pour ses publicités radio le personnage de Madame Laurent, archétype de la personne âgée éprouvant un récurrent besoin des services en question.

Chaque écran publicitaire pour Télé-Secours s'organise ainsi autour de l'évocation d'une histoire survenue à Madame Laurent. Cette dernière n'est toutefois pas fréquemment mise en scène, à la manière Leclerc : il n'y a qu'un spot où l'histoire est narrée par Madame Laurent elle-même. Dans celui-ci, l'héroïne raconte son aventure alors que l'on entend, en arrière-plan sonore, le tic-tac d'une pendule qui finit par sonner les heures.

"Hier, j'ai fait une mauvaise chute dans ma cuisine, voyez-vous. Je ne savais pas me relever. J'étais seule, comme toujours. Mais grâce à mon petit émetteur Télé-Secours, on a pu prévenir mes voisins. Et ils m'ont sauvée, très rapidement. D'ailleurs, Télé-Secours est toujours à l'écoute. »

D'ordinaire, l'histoire est plutôt racontée par un narrateur extradiégétique, plus intellectualisant, qui décharge en partie le récit de son caractère vécu et personnalisé.

Ainsi, ce spot où l'on entend de jeunes enfants faire un compte à rebours puis pousser des cris de joie, comme lorsque quelqu'un souffle les bougies d'un gâteau d'anniversaire. Ensuite, le narrateur intervient :

«Ça, c'est les proches de Madame Laurent qui est abonnée à TéléSecours. Elle a fait une mauvaise chute, et elle habite seule. Mais grâce à son pendentif, Télé-Secours a pu prévenir ses proches. Ils ont été très efficaces. À présent, elle va bien. »

Enfin, dans cet autre écran sonore, le narrateur peut même en quelque sorte se révéler "métadiégétique » dans la mesure où il n'est pas lui-même à la source de l'histoire et ne la narre pas réellement :

«-Bernard Deroever, vous êtes directeur de Télé-Secours. Alors, comment va Madame Laurent? 
- Ne vous en faites pas, elle va très bien. Grâce à Télé-Secours, elle vit toujours en sécurité, seule dans sa maison.

- Mmh, Mmh. Et elle porte toujours son émetteur Télé-Secours?

- Ah bien entendu. Et elle est en grande forme. »

La publicité à héros récurrent constitue une des tendances de l'écriture commerciale radiophonique contemporaine. À côté du cas de Madame Luarent, les auditeurs belges francophones sont ainsi aussi familiers des péripéties des Devos et Lemmens, deux joyeux drilles à l'accent bruxellois, trop heureux de fêter l'été de barbecue en barbecue pour autant qu'ils soient arrosés de sauces... de la marque DevosLemmens. Et ce cas n'est pas isolé. Mais on ne peut toutefois affirmer que le recours à des personnages récurrents et à une configuration feuilletonisante représentent le courant général de la publicité radio.

Par contre, il est manifeste que, en Belgique francophone, le caractère narratif global des écrans publicitaires radiophoniques s'est fortement affirmé depuis le tournant des années 2000. De ce point de vue, ces publicités radiophoniques s'apparentent de plus en plus au modèle que l'on rencontre dans une importante part des écrans publicitaires télévisés, qui ont eux aussi adopté la mécanique de valorisation d'un produit de marque via un mode narratif.

La représentation sonore de situations campées par des personnages, soit des mises en scène, constitue une partie du recours à ce mode d'écriture.

Tel est par exemple le cas dans une série de messages promotionnels pour les portes de garage Hormann. Ceux-ci ont en commun de représenter des personnages en situation d'action de manière telle qu'un simple léger décor sonore suffit à l'auditeur pour se représenter la scène évoquée.

Dans une d'entre elles, l'écran commence par un fort crissement de pneus, suivi du dialogue suivant :

«-Stooop!

— Mais pourquoi dois-je m'arrêter chez les voisins?

- Tu as vu leur nouvelle porte de garage?

- Ben oui, leur nouvelle porte de garage Hormann est vraiment formidable. 
- Extraordinaire, tu veux dire! Leur maison est dix fois plus belle! Moi aussi je veux une porte comme celle-là.

- Eh bien ça tombe bien. Il y a justement des Journées nationales des dealers Hormann. C'est le moment d'y aller. »

Dans une autre cas, un couple de jeunes vit un scénario plus élaboré, commençant par quelques bruits de pas étouffés :

«-Chut, ne réveille pas papa!

- Mais non, on rentre par le garage!

- Et il ne l'entendra pas?

- Mais non, cette porte Hormann ne fait absolument aucun bruit !... Ah zut, j’ai oublié les clés !

- Mais enfonce la porte!

- Petite maligne, les portes Hormann résistent à l'effraction. Il faut sonner.

- Et encore un mois privée de sorties!»

(Ils sonnent, le père surgit et rugit : «Et alors !?»)

La mise en scène de personnages en action n'est cependant pas le mode narratif radiophonique le plus manifeste dans les publicités. À l'image du média radiophonique dans son ensemble, qui a coutume à valoriser le recours au testimonial davantage qu'à la représentation de situations vécues, la publicité radio fait, elle aussi, plutôt usage de témoignages.

Le récit se manifeste alors de manière détournée, n'intervenant que via le teneur du message rapporté par un témoin de la scène.

On songera à ce propos, dans le cadre de modèles à configuration récurrente, aux publicités Carglass où un ouvrier ou un employé de la firme, selon les cas, racontent la mésaventure survenue à un client dont le pare-brise s'est fissuré, avant d'évoquer la solution trouvée par le réparateur.

Dans ce cas, en début de spot, un incipit morphologiquement récurrent, reprenant le nom, l'âge et la fonction de l'employé de la firme, sert 
de point de repère à l'auditeur, manifestant qu'il va ensuite entendre un témoignage sur un « cas » résolu par l'intervention de la société ${ }^{1}$.

Laurence, 35 ans, assistante dans un centre Carglass. "Souvent, il $y$ a des gens qui ont un petit éclat dans leur pare-brise, et ils ne veulent rien faire. Un monsieur, par exemple, l'autre jour, il veut rentrer dans son garage, il passe sur une bordure (...). »

Marjorie, 29 ans, manager d'un centre Carglass. "Normalement nous travaillons sur base d'un rendez-vous, sauf pour les cas exceptionnels. Je me souviens notamment d'un cas exceptionnel (...). »

Eddy, réparateur Carglass en déplacement. "Non, non, les gens n'ont pas toujours le temps de passer à l'atelier. Alors on prend rendezvous et on va faire le boulot chez eux ou au bureau. Tout ce qui nous faut est de pouvoir travailler à l'abri. Eh bien une fois j'ai dû me rendre chez un pâtissier (...).»

Laurent, chef d'équipe à Marignane. "Ma cliente, là, c'est pas croyable les journées qu'elle passe entre son travail, les aller-retour à l'école, les repas à préparer et j'en passe, elle a pas une minute à elle (...).»

Dans leur ensemble, ces exemples confirment l'importance prise par le narratif dans le contextualisant de la publicité radio. Bien évidemment, le récit n'est utilisé ici que comme moyen afin de valoriser le caractère promotionnel du message. Il remplit un rôle d'alerte, joue sur le référent acquis ainsi que sur la fidélisation. Il permet à l'auditeur de se repérer au sein d'un monde connu. Mais il est indéniable qu' amorcer une publicité par un récit constitue également un moyen de répondre aux attentes profondes de l'auditeur, toujours en soif d'un narratif que la radio est souvent en peine de lui livrer.

\section{Radio et récits}

Les travaux menés depuis le début des années 1990 par l'Observatoire du Récit Médiatique (ORM) de l'UCL reposent sur la conviction du rôle fondateur joué à l'heure actuelle par les récits médiatiques dans la constitution de l'identité de l'individu contemporain.

1 Les trois premiers exemples ont été diffusés en Belgique, le quatrième en France. 
S'inspirant des travaux de Paul Ricœur ${ }^{1}$, ils sont convaincus du fait que la configuration des contenus en des récits médiatiques est nécessairement suivie d'une refiguration de ces éléments narratifs par les destinataires des messages.

Un simple regard sur ce qui constitue l'essentiel du modèle énonciatif médiatique actuel ne peut que confirmer cette thèse, à l'exception peut-être toutefois d'un des univers médiatiques contemporains qui nous occupe ici : celui de la radio, où le narratif semble à l'heure actuelle peu prégnant. Et ce alors qu'il domine dans la majorité des autres médias, que ce soit dans le cadre de productions de nature fictionnelle ou dans celui de la mise en forme d'éléments liés aux mondes du réel, de l'actualité et de l'information ${ }^{2}$.

Faut-il rappeler qu'il n'en fut pas toujours ainsi, et qu'à ses débuts la radio fut également un média fortement teinté de narratif. Les débats sur la spécificité de l'écriture narrative radiophonique dans le cadre de la dramatique radio (radio drama) sont presque aussi anciens que l'existence du média lui-même.

Dès 1926, les premières réflexions à ce propos avaient été systématisées par Gabriel Germinet, alias Maurice Vinot, directeur de RadioParis, dans un ouvrage au titre explicite : Le théâtre radiophonique, mode nouveau d'expression artistique ${ }^{3}$. L'auteur y évoquait notamment les possibilités de la TSF et ses exigences de spectacle, parlant du « théâtre invisible » qu'était la radio et de l'inférence de cet état de choses sur la nature du modèle narratif radiophonique.

Si certains autres auteurs, comme René Sudre, estimeront par la suite que "l'abstraction auditive semble impuissante à constituer un art autonome comme l'avait fait l'abstraction visuelle du cinéma muet $»^{4}$, Germinet était lui convaincu du contraire, mais marquait la différence entre le «théâtre radiophoné », ou la captation de pièces de théâtre, et

1 Ricœur P., Temps et récit, Tomes I à III, Paris, Seuil 1983 à 1985.

2 La base de l'écriture littéraire fictionnelle est essentiellement narrative et il en est de même de l'écriture cinématographique et télévisuelle. Que ce soit dans les fictions télévisuelles, les clips vidéo, les programmes de télé-réalité, les magazines, les reportages, les séquences d'information ou les spots publicitaires, par exemple.

3 Paris, Étienne Chiron, 1926.

4 Sudre R., Le huitième art : mission de la radio, Paris, Julliard, 1945. 
le véritable «théâtre radiophonique » reposant sur des œuvres originales écrites pour la radio ${ }^{1}$.

La particularité de la narration radiophonique était de raconter sans montrer. Cette obligation d'agir sur l'évocation et non sur la monstration constituait incontestablement un handicap pour le média radiophonique en regard des médias relevant de l'univers du visuel. Mais elle pouvait aussi s'avérer sa chance. Comme l'évoquait Carlos Larronde dans une des préfaces du livre de Germinet, l'évocatif ouvrait à la radio des champs d'expression plus larges que ceux de la simple représentation : "Non, il ne faut pas considérer les auditeurs comme des aveugles. Ils sont autre chose. Ils sont des "sur-auditifs ». Sachons leur donner tout ce que l'ouïe, le sens subtil et intérieur par excellence, peut accueillir de lyrisme, de rêve ou d'évocation. $»^{2}$

Cet art du récit radiophonique fictionnel a profondément marqué le média. Il connut ses heures de gloire dans les années 1950, considérées comme «l'âge d'or de la création radiophonique $»^{3}$. Mais on aurait tort de confiner à l'univers de la fiction les richesses de l'écriture narrative de la radio. Malgré la lourdeur des moyens techniques à mettre en œuvre, les productions sonores des années 1930 témoignent déjà de la qualité des reportages et des documentaires radiophoniques.

À la même époque, la radio démontrait aussi son incomparable don de médiation dans la transmission des émotions lorsqu'elle recourait à l'apport testimonial de reporters vivant en temps réel des événements dramatiques. Que l'on songe, à simple titre d'exemple, au célèbre reportage de Herbert Morrison diffusé par la station WLN Radio Chicago le 6 mai 1937 lors de la catastrophe du dirigeable Hindenburg ${ }^{4}$.

Radio et récit ont ainsi été intimement liés jusqu'à l'avènement de la télévision, comme le rappelle à suffisance à elle seule l'origine

1 TODD C., « Gabriel Germinet and the "Livre d'or du théâtre radiophonique français" (1923-1935) » in : Modern and Contemporary France, University of Leeds, 2002, taylorandfrancis.metapress.com/ index/KXAUWHX35YKRHFF4.pdf

2 LARRONDE C., préface à l'édition du Théâtre Invisible, Paris, 1936.

3 HARTJE H., « Radio-plume », in : Histoires littéraires, ${ }^{\circ} 13$, janvier- mars. Paris 2003.

$4 \mathrm{http}: / /$ www.americanrhetoric.com/mp3clips/speeches/

herbmorrisonhindenburgdisaster.mp3 
des soaps operas actuellement diffusés à profusion sur les chaînes de télévision, mais qui furent portés sur les fonts baptismaux par le média radio.

À l'instar des soaps ou des feuilletons qui, pour la plupart, disparurent de ses antennes au tournant des années 1960, la radio dans son ensemble semble s'être détournée à ce moment des univers narratifs. Comme si, face aux médias de la monstration, le discursif avait quasi naturellement pris la place du narratif dans la palette des modes expressifs radiophoniques ${ }^{1}$, la radio choisissant de relever du monde de l'énonciation des idées, laissant aux médias audiovisuels celui de leur représentation. D'où le fameux adage, ressassé pendant tant d'années, selon lequel la radio annonçait, la télévision montrait et la presse écrite expliquait. On sait aujourd'hui que cet adage n'est plus d'actualité.

Le cas d'école du rapport entre narration et publicité radiophonique, envisagé en exergue de cet article, démontre que le mode d'expression par le récit a commencé à renouer avec le radiophonique. Une tendance marquante du futur de la radio résidera assurément dans un nouveau développement de ses formes narratives, qui devront tenir compte des évolutions connues par le média. Il est en effet incontestable que le style et la forme véhiculés par la dramatique radiophonique classique sont aujourd'hui non seulement dépassés mais sont devenus inaudibles par la quasi-totalité de la population, formatés par les modèles narratifs télévisuels et audiovisuels.

Comme tous les autres médias, la radio doit désormais réapprivoiser le narratif. Mais sans se contenter de remettre d'anciennes recettes en exploitation.

Un des secteurs de recherches futures sur la radio résidera certainement dans l'analyse des nouvelles caractéristiques du récit à la radio. Il devra s'intéresser aux types de récits ayant trouvé dans la radio le meilleur moyen de s'exprimer, à la manière dont ils sont élaborés et aux fonctions remplies, à l'avenir, par le narratif radiophonique.

Une question essentielle concernera les formats qu'adopteront les récits. Il sera dès lors possible de déterminer si le modèle court de la

1 Cfr. le titre du célèbre ouvrage de Patrick CHARAUDEAU Aspects du discours radiophonique (1984). 
« capsule » ou de la «pastille », présenté ci-dessus dans le cadre des spots publicitaires narrativisés, est résolument devenu celui qui convient le mieux à la construction d'histoires à diffuser à la radio. 